\title{
Universal properties of type IIB and F-theory flux compactifications at large complex structure
}

\author{
M.C. David Marsh ${ }^{a}$ and Kepa Sousa ${ }^{b, c}$ \\ ${ }^{a}$ Department of Applied Mathematics and Theoretical Physics, University of Cambridge, \\ Cambridge, CB3 0WA, U.K. \\ ${ }^{b}$ Instituto de Fisica Teorica UAM-CSIC Universidad Autonoma de Madrid, \\ Cantoblanco, 28049 Madrid, Spain \\ ${ }^{c}$ Department of Theoretical Physics and History of Science, \\ University of the Basque Country UPV/EHU, \\ 48080 Bilbao, Spain \\ E-mail: m.c.d.marsh@damtp.cam.ac.uk, kepa.sousa@csic.es
}

ABSTRACT: We consider flux compactifications of type IIB string theory and F-theory in which the respective superpotentials at large complex structure are dominated by cubic or quartic terms in the complex structure moduli. In this limit, the low-energy effective theory exhibits universal properties that are insensitive to the details of the compactification manifold or the flux configuration. Focussing on the complex structure and axio-dilaton sector, we show that there are no vacua in this region and the spectrum of the Hessian matrix is highly peaked and consists only of three distinct eigenvalues $\left(0,2 m_{3 / 2}^{2}\right.$ and $\left.8 m_{3 / 2}^{2}\right)$, independently of the number of moduli. We briefly comment on how the inclusion of Kähler moduli affect these findings. Our results generalise those of Brodie \& Marsh [1], in which these universal properties were found in a subspace of the large complex structure limit of type IIB compactifications.

KEYWORDS: Flux compactifications, Superstring Vacua

ArXIV EPRINT: 1512.08549 


\section{Contents}

1 Introduction 1

2 The low-energy theory of flux compactifications 3

2.1 Type IIB compactifications 3

2.2 F-theory compactifications 5

$\begin{array}{lll}3 & \text { Universal properties at large complex structure } & 6\end{array}$

3.1 Type IIB compactifications $\quad 6$

3.1.1 Canonical normalisation and Yukawa couplings 6

$\begin{array}{ll}\text { 3.1.2 Value of the potential and its gradient } & 7\end{array}$

3.1.3 Spectrum 8

$\begin{array}{lll}3.2 & \text { F-theory compactifications } & 10\end{array}$

$\begin{array}{lll}3.2 .1 & \text { Canonical normalisation and Yukawa couplings } & 10\end{array}$

$\begin{array}{ll}3.2 .2 & \text { Value of the potential and its gradient }\end{array}$

$\begin{array}{lll}3.2 .3 & \text { Spectrum } & 11\end{array}$

4 Conclusions $\quad 12$

\section{Introduction}

String theory can be compactified on any of a large set of permissible manifolds, each typically admitting numerous configurations of fluxes and branes. At low energies, this results in a large set of string theory-derived four-dimensional effective field theories. Unfortunately, typical examples of such theories involve a large number of fields and are very challenging to explicitly construct and solve. Statistical methods that circumvent direct enumeration of explicit solutions have proven valuable [2], however, as such methods invariably simplify the problem by making certain approximations, it is of obvious importance to delineate the corresponding regimes of applicability.

The primary assumption of statistical studies of flux compactifications is that the generalised Dirac quantisation condition on the fluxes can be ignored when the typical number of flux quanta is large. In other words, the statistical properties of the ensemble of effective theories obtained from summing over quantised fluxes are approximated by a continuous integral over these fluxes so that, schematically,

$$
\sum_{\tilde{N}} \longrightarrow \int \mathrm{d} \tilde{N},
$$

for the a priori quantised flux $\tilde{N}$. This 'continuous flux approximation' is of key importance in the derivation of the index of supersymmetric flux vacua [2], as well as the subsequent statistical studies $[3-5]$. 
Building on the continuous flux approximation, it was pointed out in [5] that the distributions of certain matrices that appear in the low-energy effective theory, should - for sufficiently complicated compactification manifolds with many moduli - be well approximated by models from random matrix theory (RMT). This idea was further expounded upon in [6], where it was shown that in such 'random supergravities', typical de Sitter critical points are exceedingly unlikely to be metastable vacua. The celebrated statistical universality of RMT models suggests that these results should be roughly independent of the microscopic details of the distributions of the couplings in the theory.

However, when the distributions of the couplings are substantially modified, the appropriate model to describe the ensemble of effective theories may be different, and the nature of these 'universal' limits could change. For instance, supergravity theories with multiple sectors appear frequently in string compactifications and may support de Sitter vacua at a much higher frequency than typical critical points in random supergravity [6-9]. Further discussions of random supergravities and string compactifications can be found in [10-15].

Recently, it was shown that the low-energy theory of type IIB flux compactifications close to a large complex structure (LCS) point satisfies certain universal properties that are independent of the number of moduli, the details of compactification geometry, or the detailed flux choice [1]. Specifically, upon restricting to the complex structure and axiodilaton sector of compactifications with $h^{1,2}$ complex structure moduli, it was shown that an $h^{1,2}+3$ real-dimensional subspace of the LCS limit contains no supersymmetric vacua and that the spectrum of the Hessian at any point in this region is given by,

$$
m_{i}^{2}= \begin{cases}0 & i=1, \ldots, h^{1,2} \\ 2 m_{3 / 2}^{2} & i=h^{1,2}+1, \ldots, 2 h^{1,2}+1, \\ 8 m_{3 / 2}^{2} & i=2\left(h^{1,2}+1\right)\end{cases}
$$

where $m_{3 / 2}=e^{K / 2}|W|$ denotes the gravitino mass. These results are in stark contrast to the expectations derived from the continuous flux approximation and the proposed RMT models (which predict a smooth spectrum), and thus serve to indicate a limit of applicability of these statistical techniques and approximations. Moreover, it was shown in [1] that the scalar potential in this region of the moduli space is too steep to support inflation, thus presenting a significant obstacle for realising inflation at large complex structure.

In this paper, we extend the results of [1] to the entire $2\left(h^{1,2}+1\right)$ dimensional moduli space for flux compactifications of type IIB string theory at large complex structure. We show that as long as the superpotential is dominated by terms cubic in the complex structure moduli, the spectrum of the Hessian is given by equation (1.2).

The generality of this conclusion is intimately related to the unbounded relative growth of a single period of the compactification manifold close to a LCS point. Our proof relies heavily on the universal form of the orthonormal-frame Yukawa couplings in the LCS limit $[4,16,17]$, from which all the relevant properties of the low-energy theory can be derived.

Flux compactifications of F-theory are particularly interesting since the topological complexity of elliptically fibered Calabi-Yau four-folds greatly exceeds that of three-folds, 
and one may expect that this should lead to a very large number of flux vacua arising from F-theory compactifications [18]. Here, we show that our results for type IIB flux compactifications generalise in a straightforward way to a corresponding LCS limit of F-theory compactifications. In particular, we show that in regions of the moduli space where the superpotential is dominated by terms quartic in the complex structure moduli, there are no vacua, and the spectrum of complex structure deformations is again given by equation (1.2), mutatis mutandis.

Finally, we would like to emphasise that our derivation neglects possible interactions of the axio-dilaton and complex structure moduli with other degrees of freedom, such as the Kähler moduli. Including such interactions will generically affect the spectra, unless the additional sectors can be integrated out or consistently truncated while preserving supersymmetry [19-25]. In string compactifications, the form of the interactions with the Kähler sector is partly determined by the corresponding moduli stabilisation mechanism, which we here don't specify. We show however that the effect of including the tree-level couplings with the Kähler moduli, which appear in a no-scale Kähler potential but not in the superpotential, is to induce simple shifts in the spectrum (1.2). We expect this effect to be relevant also for fully stabilised compactifications.

This paper is organised as follows: in section 2 we review the form of the $\mathcal{N}=1$ supergravities arising in the low-energy limit of flux compactifications of type IIB string theory and F-theory. Section 3 contains our computations and results, and section 4 our conclusions.

\section{The low-energy theory of flux compactifications}

In this section, we briefly review the elements of the four-dimensional effective theories arising from flux compactifications of type IIB string theory and F-theory [26, 27].

\subsection{Type IIB compactifications}

The low-energy spectrum of compactifications of type IIB supergravity on the orientifold $\tilde{M}_{3}$ of the Calabi-Yau three-fold $M_{3}$ include the axio-dilaton, $\tau=C_{0}+i e^{-\phi}$, the complex structure moduli, $\phi^{i}$, where $i=1, \ldots, h_{-}^{1,2}\left(\tilde{M}_{3}\right)$, and the Kähler moduli, $T^{\alpha}$, where $\alpha=$ $1, \ldots, h_{+}^{1,1}\left(\tilde{M}_{3}\right) .{ }^{1}$ We are interested in compactifications in which integrally quantised RR $\left(F_{3}\right)$ and NS-NS $\left(H_{3}\right)$ fluxes wrap some non-trivial three-cycles of $M_{3}$,

$$
\frac{1}{(2 \pi)^{2} \alpha^{\prime}} \int_{A^{I}, B_{I}} F_{3}=\vec{N}_{\mathrm{RR}} \in \mathbb{Z}^{2\left(h^{1,2}+1\right)}, \quad \frac{1}{(2 \pi)^{2} \alpha^{\prime}} \int_{A^{I}, B_{I}} H_{3}=\vec{N}_{\mathrm{NS}-\mathrm{NS}} \in \mathbb{Z}^{2\left(h^{1,2}+1\right)} .
$$

Here $\left(A^{I}, B_{I}\right)$ with $I=0, \ldots, h^{1,2}\left(M_{3}\right)$ denotes an integral and symplectic homology basis of $H_{3}\left(M_{3}, \mathbb{Z}\right)$, satisfying,

$$
A^{I} \cap B_{J}=\delta_{J}^{I}, \quad A^{I} \cap A^{J}=B_{I} \cap B_{J}=0 .
$$

\footnotetext{
${ }^{1}$ We here ignore the possible existence of axion multiplets $G^{\alpha}$, with $\alpha=1, \ldots, h_{-}^{1,1}\left(\tilde{M}_{3}\right)$, that are not lifted by the fluxes.
} 
The RR and NS-NS three-form flux is conveniently expressed in the combination $G_{3}=$ $F_{3}-\tau H_{3}$, and we denote the corresponding complexified flux vector by,

$$
\vec{N}=\Sigma\left(\begin{array}{l}
\int_{A^{I}} G_{3} \\
\int_{B_{I}} G_{3}
\end{array}\right), \quad \text { with } \quad \Sigma=\left(\begin{array}{rr}
0 & \mathbb{1} \\
-\mathbb{1} & 0
\end{array}\right) .
$$

The Kähler potential of the low-energy effective supergravity of type IIB flux compactifications is given to leading order in $g_{s}$ and $\alpha^{\prime}$ by,

$$
K=-\ln \left(i \int_{M_{3}} \Omega \wedge \bar{\Omega}\right)-\ln (-i(\tau-\bar{\tau}))-2 \ln \mathcal{V} .
$$

Here, $\mathcal{V}$ denotes the Kähler moduli dependent compactification volume and $\Omega$ denotes the complex structure moduli dependent holomorphic three-form of $M_{3}$.

The non-trivial fluxes induce a complex structure and axio-dilaton dependent superpotential [28],

$$
W=\int_{M_{3}} G_{3} \wedge \Omega
$$

The complex structure dependence is conveniently expressed through the period vector,

$$
\vec{\Pi}=\left(\begin{array}{c}
\int_{A^{I}} \Omega \\
\int_{B_{I}} \Omega
\end{array}\right)
$$

where $z^{I}=\int_{A^{I}} \Omega$ are homogenous coordinates on the complex structure moduli space. The corresponding inhomogeneous coordinates, e.g. $\phi^{i}=-i z^{i} / z^{0}$ for $z^{0} \neq 0$, defines the corresponding complex structure moduli fields for $i=1, \ldots, h^{1,2}$. Upon setting $z^{0}=1$, the period vector is given by,

$$
\vec{\Pi}=\left(\begin{array}{c}
1 \\
i \phi^{i} \\
2 F-\phi^{j} F_{j} \\
-i F_{i}
\end{array}\right),
$$

where $F\left(\phi^{i}\right)$ denotes the holomorphic $\mathcal{N}=2$ prepotential of the underlying special geometry of the complex structure moduli space.

The functional form of $F\left(\phi^{i}\right)$ in the neighbourhood of a large complex structure point located at $\operatorname{Re}\left(\phi^{i}\right) \rightarrow \infty$, is given by,

$$
F=\frac{i}{6} \kappa_{i j k} \phi^{i} \phi^{j} \phi^{k}+\frac{1}{2} \kappa_{i j} \phi^{i} \phi^{j}+i \kappa_{i} \phi^{i}+\frac{1}{2} \kappa_{0}+I
$$

where $I$ denotes exponentially suppressed instanton contributions that will not be important to our discussion. The classical expansion coefficients are given by intersections of the mirror dual Calabi-Yau three-fold $M_{3}^{\mathrm{d}}$ [29-31] (see also [32]). For historical reasons, the coefficients $\kappa_{i j k}$ are called (the classical) Yukawa couplings [33].

The orientifold action projects out some of the moduli from the spectrum and reduces the amount of supersymmetry to $\mathcal{N}=1$ in $d=4$. The complex structure dependent part 
of the Kähler potential may now be written as,

$$
\begin{aligned}
K_{\text {c.s. }} & =-\ln \left(i \int_{M_{3}} \Omega \wedge \bar{\Omega}\right)=-\ln \left(i \vec{\Pi}^{\dagger} \Sigma \vec{\Pi}\right) \\
& =-\ln \left(\frac{1}{6} \kappa_{i j k}(\phi+\bar{\phi})^{i}(\phi+\bar{\phi})^{j}(\phi+\bar{\phi})^{k}-2 \operatorname{Im}\left(\kappa_{0}\right)\right) .
\end{aligned}
$$

When,

$$
Y\left(\phi^{i}+\bar{\phi}^{i}\right) \equiv \frac{1}{6} \kappa_{i j k}(\phi+\bar{\phi})^{i}(\phi+\bar{\phi})^{j}(\phi+\bar{\phi})^{k} \gg 2 \operatorname{Im}\left(\kappa_{0}\right),
$$

the Kähler potential is well approximated by,

$$
K_{\text {c.s. }} \approx-\ln \left(Y\left(\phi^{i}+\bar{\phi}^{i}\right)\right)
$$

For any given flux configuration with $N \equiv(\vec{N})_{h^{1,2}+2} \neq 0$, it follows from the LCS expansion (2.7) that the superpotential (2.4) for sufficiently large values of the moduli is approximated by,

$$
W=\vec{N} \cdot \vec{\Pi} \approx-i \frac{N}{6} \kappa_{i j k} \phi^{i} \phi^{j} \phi^{k}+\mathcal{O}\left(\phi^{2}\right) .
$$

Here we have used that the cubic terms arise from a single cycle and hence appear in the superpotential multiplied by a single complexified flux component, $N$.

In this paper we only consider the generic case with $N \neq 0$. For any non-trivial flux configuration, we can choose the inhomogeneous coordinates on moduli space in such a way that the $z^{0}$ variable corresponds to a cycle with non-vanishing flux, and hence $N \neq 0$. Clearly, for a given flux configuration with some vanishing flux numbers, there will in general exist LCS points for which the period supporting the cubic terms in the moduli does not appear in the flux superpotential and our results do not apply.

In sum, in this paper we consider the type IIB flux compactifications that are described by the four-dimensional $\mathcal{N}=1$ supergravity with,

$$
K=-\ln (Y)-\ln (-i(\tau-\bar{\tau})), \quad W=-i \frac{N}{6} \kappa_{i j k} \phi^{i} \phi^{j} \phi^{k},
$$

where $Y$ is defined by equation (2.9). We will for now ignore Kähler moduli as these do not appear in the flux superpotential, but we will briefly comment on their inclusion in section 4 .

\section{$2.2 \quad$ F-theory compactifications}

Compactifications of F-theory can be viewed as a generalisation of type IIB compactifications to include non-trivial axio-dilaton profiles in the internal space. In this description, $\tau$ is identified with the complex structure modulus of an elliptic curve fibered over a compact three dimensional base manifold (that need not be Calabi-Yau). In supersymmetric compactifications, the resulting four-fold, $X$, is Calabi-Yau and the four-dimensional $\mathcal{N}=1$ low-energy supergravity for the complex structure moduli is described by,

$$
K=-\ln \left(\int_{X} \Omega_{4} \wedge \bar{\Omega}_{4}\right), \quad W=\int_{X} G_{4} \wedge \Omega_{4} .
$$


The complex structure moduli, $\phi^{i}$ with $i=1, \ldots, h^{3,1}(X)$, may in analogy with type IIB compactification be defined from the periods of the four-fold. In the large complex structure limit of manifold $X$, one of the periods, say $\Pi_{\max }$, grows as,

$$
\Pi_{\max } \sim \kappa_{i j k l} \phi^{i} \phi^{j} \phi^{k} \phi^{l}
$$

while other periods grow at most as $\sim \mathcal{O}\left(\phi^{3}\right)$. Here $\kappa_{i j k l}$ denote the classical intersection numbers of the divisors of the mirror dual four-fold, as discussed in [34-37].

We will consider flux compactifications of F-theory in which the four-form flux $G_{4}$ on the corresponding cycle does not vanish. The superpotential and Kähler potential are then well-approximated by,

$$
K=-\ln \left(\frac{1}{4 !} \kappa_{i j k l}(\phi+\bar{\phi})^{i}(\phi+\bar{\phi})^{j}(\phi+\bar{\phi})^{k}(\phi+\bar{\phi})^{l}\right), \quad W=N \kappa_{i j k l} \phi^{i} \phi^{j} \phi^{k} \phi^{l},
$$

at large complex structure. We note that as the Hodge numbers of elliptically fibered Calabi-Yau four-folds tend to be quite large $\mathcal{O}\left(h^{3,1}\right) \sim 10^{5}$, the superpotential of equation (2.15) is in general a nontrivial function containing up to $\sim 10^{18}$ terms. $^{2}$ We will nevertheless show that it posses enough structure to be entirely computable with rudimentary techniques.

\section{Universal properties at large complex structure}

In this section we extend the results of [1] by showing that the regions of the moduli space where the low-energy theory is well-described by equation (2.12) or equation (2.15) exhibit certain universal properties, such as simple expressions for the gradient of the scalar potential and the spectrum of the Hessian matrix.

Our method is very similar to that of [17] and is quite straight-forward: for a given point $p$ in the moduli space, we make a coordinate transformation $\phi^{i} \rightarrow \varphi^{a}$ to canonically normalise the Kähler metric. In these new coordinates, the Yukawa couplings satisfy certain non-trivial relations which we use to derive the explicit form of the F-terms, the spectrum of the canonically normalised fields, and the inflationary slow-roll parameters.

\subsection{Type IIB compactifications}

\subsubsection{Canonical normalisation and Yukawa couplings}

We begin by considering a general point $p$ in the moduli space for which (2.12) provides a good approximation of the low-energy theory. The Kähler metric is then real and in the standard coordinates $\left(\tau(p), \phi^{i}(p)\right)$ given by,

$$
\begin{aligned}
K_{\tau \bar{\tau}} & =-\frac{1}{(\tau-\bar{\tau})^{2}}, \\
K_{i \bar{\tau}} & =K_{\tau \bar{j}}=0 \\
K_{i \bar{j}} & =-\frac{\kappa_{i j k}}{Y}\left(\phi^{k}+\bar{\phi}^{k}\right)+\frac{\kappa_{i l m} \kappa_{j n p}}{4 Y^{2}}\left(\phi^{l}+\bar{\phi}^{l}\right)\left(\phi^{m}+\bar{\phi}^{m}\right)\left(\phi^{n}+\bar{\phi}^{n}\right)\left(\phi^{p}+\bar{\phi}^{p}\right) .
\end{aligned}
$$

\footnotetext{
${ }^{2}$ Our results are equally applicable for the case of sparse Yukawa couplings.
} 
The metric on the complex structure moduli space can locally at $p$ be brought to the canonically normalised form by a change of variables. Here, we will define $\mathcal{G} \in \operatorname{GL}\left(h_{-}^{1,2}, \mathbb{R}\right)$ to be the transformation, $\varphi^{a}=\mathcal{G}_{i}^{a} \phi^{i}$, that canonically normalises the metric, and aligns the moduli vacuum expectation value (vev) with the first coordinate axis, such that $\varphi^{a}=\varphi_{0} \delta_{1}^{a}$. The Kähler potential and the function $Y$ defined through equation (2.9) are scalars and hence invariant under this coordinate transformation. The Yukawa couplings transform as,

$$
\kappa_{a b c}=\left(\mathcal{G}^{-1}\right)^{i}{ }_{a}\left(\mathcal{G}^{-1}\right)^{j}{ }_{b}\left(\mathcal{G}^{-1}\right)^{k}{ }_{c} \kappa_{i j k} .
$$

In the new coordinate system, where $K_{a \bar{b}}=\delta_{a \bar{b}}$, equation (3.3) reads,

$$
\delta_{a b}=-\frac{\kappa_{a b 1}}{Y} x+\frac{\kappa_{a 11} \kappa_{b 11}}{4 Y^{2}} x^{4}
$$

where we have denoted $\varphi_{0}=x / 2+i y / 2$, and dropped the bar on the indices for the real Kähler metric. Note that from the definition (2.9), $Y=\frac{1}{6} \kappa_{111} x^{3}$. Equation (3.5) has several non-trivial implications for the orthonormal-frame Yukawa couplings,

$$
\kappa_{111}=\frac{2}{\sqrt{3}} Y, \quad \kappa_{11 a^{\prime}}=0, \quad \kappa_{1 a^{\prime} b^{\prime}}=-\frac{1}{\sqrt{3}} \delta_{a^{\prime} b^{\prime}} Y,
$$

in agreement with the results found in $[4,16]$ for models with a single complex structure modulus. Here $a^{\prime}, b^{\prime} \in\left\{2, \ldots, h_{-}^{1,2}\right\}$. Crucially important for our following derivations is that the Yukawa couplings drop out of first equation of (3.6), which then restricts the value of the real component of $\varphi_{0}$,

$$
x^{2}=3 .
$$

Since $x>0$, we find that in these coordinates the real part of the modulus vev is uniquely fixed, $\varphi_{0}=\sqrt{3} / 2+i y / 2$, and $\kappa_{a^{\prime} b^{\prime} 1}=-\frac{\kappa_{111}}{2} \delta_{a^{\prime} b^{\prime}}$.

The Yukawa couplings $\kappa_{a^{\prime} b^{\prime} c^{\prime}}$ with no index along the ' 1 '-direction are not constrained by the canonical normalisation condition, however, we will see these coefficients do not appear in the expressions for the gradient vector or the Hessian matrix.

The canonical normalisation of the axio-dilaton is achieved by the vielbein, $e_{0}^{\tau}=i(\tau-$ $\bar{\tau})$, and then we may write the canonically normalised fields as $\varphi^{A}$ with $A=0$ corresponding to the normalised axio-dilaton, and $A=a=1, \ldots, h_{-}^{1,2}$ for the complex structure moduli.

\subsubsection{Value of the potential and its gradient}

It is well known from mirror symmetry that the Kähler potential for the complex structure moduli close to the LCS point is of 'no-scale type' satisfying $K_{a} K^{a}=3$. This follows directly from that the function $Y$ of (2.9) is a homogeneous function of degree 3 in the moduli, and is here explicitly evident as, in terms of the canonically normalised fields,

$$
K_{a} \equiv \partial_{\varphi^{a}} K=-\frac{1}{2} \kappa_{a 11} \frac{x^{2}}{Y}=-\sqrt{3} \delta_{a}^{1}
$$

so that indeed $K_{a} K^{a}=K_{a} K_{\bar{a}} \delta^{a \bar{a}}=3$. We note that $K_{a}$ is aligned with the real axis of the complex $\varphi^{1}$ plane. 
An interesting property of the theory defined by (2.12) is that while $\partial_{a} W \neq 0$ and $K_{a} W K^{a} \bar{W}=3|W|^{2}$, we still find that the squared magnitude of the covariant derivative $F_{a}=D_{a} W=\left(\partial_{a}+K_{a}\right) W$, satisfies $F_{a} \bar{F}^{a}=3|W|^{2}$. To see this, we note that equation (3.6) implies that,

$$
\partial_{a} W=\frac{3}{\varphi_{0}} W \delta_{a}^{1}
$$

We then have that,

$$
F_{a}=\frac{\sqrt{3}}{\varphi_{0}} W \delta_{a}^{1}\left(\sqrt{3}-\varphi_{0}\right)=\sqrt{3} W \delta_{a}^{1}\left(\frac{\varphi_{0}^{*}}{\varphi_{0}}\right)
$$

and $F_{a} \bar{F}^{a}=3|W|^{2}$. The axio-dilaton $\mathrm{F}$-term is perhaps easiest found by noting that $D_{\tau} N=K_{\tau} N^{*}$ so that $F_{\tau} \bar{F}^{\tau}=|W|^{2}$.

In sum, for the canonically normalised fields the F-terms are given by,

$$
F_{A}=\left(\begin{array}{c}
F_{0} \\
F_{a}
\end{array}\right)=\left(\begin{array}{c}
-i\left(\frac{N^{*}}{N}\right) W \\
\sqrt{3}\left(\frac{\varphi_{0}^{*}}{\varphi_{0}}\right) W \delta_{a}^{1}
\end{array}\right) .
$$

Consequently, at a point $p$ in the moduli space for which the Kähler potential and superpotential are given by equations (2.12), the magnitude of the F-terms and the scalar potential are given by,

$$
F_{A} \bar{F}^{A}=4|W|^{2} \quad \Longrightarrow \quad V=e^{K}|W|^{2} .
$$

As we have shown that the second equation in (3.12) holds at a generic point at large complex structure, the gradient can now be found through simple differentiation,

$$
\partial_{A} V=e^{K} F_{A} \bar{W}
$$

Near the LCS limit and with $N \neq 0$, the superpotential and the F-terms cannot vanish. Thus, the scalar potential has no critical points in this regime.

\subsubsection{Spectrum}

The Hessian matrix derived from the potential (3.12) is given by,

$$
\mathcal{H}=\left(\begin{array}{cc}
\nabla_{A \bar{B}} V & \nabla_{A B} V \\
\nabla_{\bar{A} \bar{B}} V & \nabla_{\bar{A} B} V
\end{array}\right)=e^{K}\left(\begin{array}{cc}
K_{A \bar{B}}|W|^{2}+F_{A} \bar{F}_{\bar{B}} & \bar{W} Z_{A B} \\
W \bar{Z}_{\bar{A} \bar{B}} & K_{\bar{A} B}|W|^{2}+\bar{F}_{\bar{A}} F_{B}
\end{array}\right),
$$

where $Z_{A B}=D_{B} F_{A}=\partial_{B} F_{A}+K_{B} F_{A}-\Gamma_{A B}^{C} F_{C}$. Thus, to find the spectrum in the entire LCS limit, we need only to find the symmetric complex tensor $Z_{A B}$. The $A=B=0$ component vanishes, as $Z_{\tau \tau}=0$ due to the linear appearance of $\tau$ in $W$ together with the simple form of the axio-dilaton Kähler potential. The mixed components are given by,

$$
Z_{\tau a}=K_{\tau} \frac{N^{*}}{N} F_{a} \quad \Longrightarrow \quad Z_{0 a}=e_{0}^{\tau} Z_{\tau a}=-i \sqrt{3}|W| e^{i \theta} \delta_{a}^{1},
$$


where we have defined the phase, ${ }^{3}$

$$
\theta=\arg \left(W\left(\frac{N^{*}}{N}\right)\left(\frac{\varphi_{0}^{*}}{\varphi_{0}}\right)\right) .
$$

The remaining components can be determined from the identity,

$$
Z_{i j}=-(\tau-\bar{\tau}) e^{K_{\text {c.s. }}} \kappa_{i j} \bar{k}_{\bar{\tau}} \bar{Z}_{\bar{\tau} \bar{k}},
$$

as reviewed in [1]. For canonically normalised fields this expression reads,

$$
Z_{a b}=i e^{K_{\text {c.s. }}} \kappa_{a b}{ }_{a b}^{\bar{c}} \bar{Z}_{\overline{0} \bar{c}}=-\frac{\sqrt{3}}{Y} \kappa_{1 a b}|W| e^{-i \theta},
$$

and the tensor $Z_{A B}$ can be expressed in matrix representation as,

$$
Z_{A B}=\left(\begin{array}{ccc}
Z_{00} & Z_{01} & Z_{0 b^{\prime}} \\
Z_{10} & Z_{11} & Z_{1 b^{\prime}} \\
Z_{a^{\prime} 0} & Z_{a^{\prime} 1} & Z_{a^{\prime} b^{\prime}}
\end{array}\right)=|W|\left(\begin{array}{ccc}
0 & -\sqrt{3} i e^{i \theta} & 0 \\
-\sqrt{3} i e^{i \theta} & -2 e^{-i \theta} & 0 \\
0 & 0 & \delta_{a^{\prime} b^{\prime}} e^{-i \theta}
\end{array}\right)
$$

We are now ready to deduce the spectrum of the axio-dilaton and complex structure moduli sector at LCS. First, we note that the $\left(a^{\prime}, b^{\prime}\right)$ directions have no cross-terms with the axio-dilaton or the ' 1 '-direction, and hence decouple. The Hessian matrix in this sector is given by $h_{-}^{1,2}-1$ copies of the same $2 \times 2$ matrix,

$$
\mathcal{H}_{\perp}=e^{K}|W|^{2}\left(\begin{array}{cc}
1 & e^{-i\left(\theta+\theta_{W}\right)} \\
e^{i\left(\theta+\theta_{W}\right)} & 1
\end{array}\right),
$$

where $\theta_{W}=\arg (W)$, and the corresponding eigenvalues are given by,

$$
m_{a^{\prime} \pm}^{2}=(1 \pm 1) m_{3 / 2}^{2},
$$

for $a^{\prime}=2, \ldots, h_{-}^{1,2}$.

The remaining eigenvalues and eigenvectors are perhaps easiest found by first noting that the F-terms (3.11) satisfy the equation,

$$
Z_{A B} \bar{F}^{B}=3 \bar{W} F_{A},
$$

with $Z_{A B}$ given by equation (3.19). The Hessian matrix then has the following eigenvectors and associated eigenvalues,

$$
v_{F \pm}=\left(\begin{array}{c}
e^{-i \theta_{W}} F^{\bar{B}} \\
\pm e^{i \theta_{W}} \bar{F}^{B}
\end{array}\right), \quad m_{F \pm}^{2}=(5 \pm 3) m_{3 / 2}^{2} .
$$

The final eigenvectors correspond to linear combinations of the ' 0 ' and ' 1 ' directions that are perpendicular to the vectors $v_{F \pm}$. These can be written in the form,

$$
v_{h \pm}=\left(\begin{array}{c}
e^{-i \theta_{W}} h^{\bar{B}} \\
\pm e^{i \theta_{W}} \bar{h}^{B}
\end{array}\right)
$$

\footnotetext{
${ }^{3}$ Note that these phases are related by the identity, $W / \bar{W}=-\left(N / N^{*}\right)\left(\varphi_{0} / \varphi_{0}^{*}\right)^{3}$.
} 
where $\bar{h}^{B} F_{B}=0$ and,

$$
Z_{A B} \bar{h}^{B}=\bar{W} h_{A}
$$

Explicitly we find,

$$
h_{A}=\left(\begin{array}{c}
-\sqrt{3} i\left(\frac{N^{*}}{N}\right)^{1 / 2}\left(\frac{\varphi_{0}}{\varphi_{0}^{*}}\right)^{3 / 2} \\
-\left(\frac{N}{N^{*}}\right)^{1 / 2}\left(\frac{\varphi_{0}}{\varphi_{0}^{*}}\right)^{1 / 2}
\end{array}\right)=\exp \left(\frac{i}{2}\left(\theta_{W}-\theta\right)\right)\left(\begin{array}{c}
\sqrt{3} i e^{2 i \theta} \\
-\delta_{a}^{1}
\end{array}\right),
$$

with the eigenvalues of the Hessian matrix,

$$
m_{h \pm}^{2}=(1 \pm 1) m_{3 / 2}^{2} .
$$

Thus, by equations (3.21), (3.23) and (3.27), we have shown that, for the entire region of the LCS expansion in which the theory is well-approximated by (2.12), the spectrum is given by equation (1.2). This is the main result of this paper.

We close this section by commenting on the spectrum of the $2\left(h_{-}^{1,2}+1\right)$ dimensional hermitian matrix $\mathcal{M}$, which is constructed as,

$$
\mathcal{M}=e^{\frac{K}{2}}\left(\begin{array}{cc}
0 & Z_{A B} e^{-i \theta_{W}} \\
\bar{Z}_{\bar{A} \bar{B}} e^{i \theta_{W}} & 0
\end{array}\right)
$$

The matrix $\mathcal{M}$ governs the non-supersymmetric critical point equation [5], and its eigenvalues determine the spectrum of fermion masses at vacua of the scalar potential and the supersymmetric contribution to the scalar masses. From equation (3.19), we find that its spectrum is given by,

$$
\lambda_{i \pm}= \begin{cases} \pm m_{3 / 2} & i=1, \ldots, h_{-}^{1,2} \\ \pm 3 m_{3 / 2} & i=h_{-}^{1,2}+1\end{cases}
$$

which again generalises the findings of [1].

\subsection{F-theory compactifications}

The computation of the values of the potential, its gradient and the spectrum of F-theory compactifications that are well-described by $(2.15)$ proceed very similarly to the type IIB case. This is not surprising, as equation (2.15) can be viewed as a special case of (2.12) with negligible RR-flux on the cycle supporting a period with cubic dependence on the complex structure moduli, upon identification of the axio-dilaton as an additional complex structure modulus. However, the computation of the properties of the low-energy theory in F-theory is perhaps even more transparent than the type IIB case, as we now show.

\subsubsection{Canonical normalisation and Yukawa couplings}

The Kähler metric derived from equation (2.15) is given by,

$$
\begin{aligned}
K_{i j}= & -12 \frac{\kappa_{i j k l}\left(\phi+\phi^{*}\right)^{k}\left(\phi+\phi^{*}\right)^{l}}{\kappa_{\text {mnop }}\left(\phi+\phi^{*}\right)^{m}\left(\phi+\phi^{*}\right)^{n}\left(\phi+\phi^{*}\right)^{o}\left(\phi+\phi^{*}\right)^{p}} \\
& +16 \frac{\kappa_{i k l m} \kappa_{\text {jnop }}\left(\phi+\phi^{*}\right)^{k}\left(\phi+\phi^{*}\right)^{l}\left(\phi+\phi^{*}\right)^{m}\left(\phi+\phi^{*}\right)^{n}\left(\phi+\phi^{*}\right)^{o}\left(\phi+\phi^{*}\right)^{p}}{\left(\kappa_{\text {qrst }}\left(\phi+\phi^{*}\right)^{q}\left(\phi+\phi^{*}\right)^{r}\left(\phi+\phi^{*}\right)^{r}\left(\phi+\phi^{*}\right)^{t}\right)^{2}} .
\end{aligned}
$$


Again, the Kähler metric is real and can locally be brought into canonical form by a $\mathcal{G} \in \mathrm{GL}\left(h^{3,1}(X), \mathbb{R}\right)$ transformation acting as $\varphi^{a}=\mathcal{G}_{i}^{a} \phi^{i}$, where $i$ and $a$ now run from 1 to $h^{3,1}(X)$. As in section 3.1.1, we take the transformation $\mathcal{G}$ to rotate the field vevs to align with the first coordinate axis, $\varphi^{a}=\varphi_{0} \delta_{1}^{a}$.

Expressed in the canonically normalised coordinates, the metric gives rise to the following non-trivial relations,

$$
x^{2}=4, \quad \kappa_{a^{\prime} 111}=0, \quad \kappa_{a^{\prime} b^{\prime} 11}=-\frac{\kappa_{1111}}{3} \delta_{a^{\prime} b^{\prime}} .
$$

for $a^{\prime}, b^{\prime} \in\left\{2, h^{3,1}(X)\right\}$. The first equation of (3.31) implies that $0<x=\varphi_{0}+\varphi_{0}^{*}=2$.

\subsubsection{Value of the potential and its gradient}

The Kähler potential of F-theory compactifications at LCS is given by the logarithm of a function that is quartic in the moduli vevs - not cubic as in the type IIB case - and hence these compactifications are not no-scale at large complex structure. In canonically normalised coordinates, we see this explicitly as $K_{a}=-2 \delta_{a}^{1}$ and $K_{a} K^{a}=4$. Using (2.15) and (3.31), we find the F-terms,

$$
F_{a}=W\left(\frac{4}{\varphi_{0}}-2\right) \delta_{a}^{1}=2 W\left(\frac{\varphi_{0}^{*}}{\varphi_{0}}\right) \delta_{a}^{1},
$$

so that $F_{a} \bar{F}^{a}=4|W|^{2}$. The values of the potential and its gradient are given by,

$$
V=e^{K}|W|^{2}, \quad \partial_{a} V=e^{K} F_{a} \bar{W},
$$

just as equations (3.12) and (3.13) for the type IIB compactifications.

\subsubsection{Spectrum}

The Hessian matrix of the system described by (2.15) is given by (3.14). Thus, we need only to compute $Z_{a b}=D_{a} F_{b}=\partial_{a} F_{b}+K_{a} F_{b}-\Gamma_{a b}^{c} F_{c}$. The relevant field space Christoffel symbols are very simple,

$$
\Gamma_{a b}^{1}=-K_{a b} .
$$

Again using that $2 / \varphi_{0}-1=\varphi_{0}^{*} / \varphi_{0}$, we express the components of $Z_{a b}$ as,

$$
\begin{aligned}
Z_{11} & =3 W\left(\frac{\varphi_{0}^{*}}{\varphi_{0}}\right)^{2} \\
Z_{1 b^{\prime}} & =0 \\
Z_{a^{\prime} b^{\prime}} & =-\delta_{a^{\prime} b^{\prime}} W\left(\frac{\varphi_{0}^{*}}{\varphi_{0}}\right)^{2} .
\end{aligned}
$$

The Hessian matrix is now simply given by $h^{3,1}(X)$ decoupled $2 \times 2$ matrices. The ' 1 'direction is special and the corresponding elements of the Hessian matrix are given by,

$$
\mathcal{H}_{F}=e^{K}\left(\begin{array}{cc}
\nabla_{1 \overline{1}} V & \nabla_{11} V \\
\nabla_{\overline{1} \overline{1}} V & \nabla_{\overline{1} 1} V
\end{array}\right)=e^{K}|W|^{2}\left(\begin{array}{cc}
5 & 3\left(\frac{\varphi_{0}^{*}}{\varphi_{0}}\right)^{2} \\
3\left(\frac{\varphi_{0}}{\varphi_{0}^{*}}\right)^{2} & 5
\end{array}\right),
$$


with the eigenvalues,

$$
m_{F_{ \pm}}^{2}=(5 \pm 3) m_{3 / 2}^{2} .
$$

The remaining eigenvalues of the Hessian are given by $h^{3,1}(X)-1$ copies of the spectrum of the $2 \times 2$ matrix,

$$
\mathcal{H}_{\perp}=e^{K}|W|^{2}\left(\begin{array}{cc}
1 & -\left(\frac{\varphi_{0}^{*}}{\varphi_{0}}\right)^{2} \\
-\left(\frac{\varphi_{0}}{\varphi_{0}^{*}}\right)^{2} & 1
\end{array}\right),
$$

giving,

$$
m_{a^{\prime} \pm}^{2}=(1 \pm 1) m_{3 / 2}^{2} .
$$

Thus, in sum, the spectrum of complex structure moduli of F-theory compactifications at LCS, as described by the low-energy theory (2.15), is exactly given by equation (1.2) upon substituting $h_{-}^{1,2}\left(\tilde{M}_{3}\right) \rightarrow h^{1,3}(X)$.

\section{Conclusions}

We have shown, in generalisation of the results of [1], that flux compactifications close to large complex structure points exhibit universal properties that are independent of the underlying details of the compactification. In the regions of the moduli space where the flux superpotentials of type IIB and F-theory compactifications are dominated by cubic or quartic terms in the complex structure moduli, respectively, the energy density is positive definite, there are no vacua, and the spectrum of the Hessian consists merely of three distinct eigenvalues.

These results should be contrasted with the expectations from the continuous flux approximation (1.1), which for large values of the D3-tadpole predicts an independence of the distribution of the superpotential and the F-terms at each point in the moduli space (cf. e.g. section 5.4 of [1]). Here we find that the magnitude of the F-terms is completely determined by the value of $|W|$ (cf. equation (3.12)), and consequently, the continuous flux approximation does not apply. 'Eigenvalue repulsion' and hence a spectrum with nondegenerate eigenvalues is a key feature of random matrix models. We here find that the spectrum typically consists of highly degenerate eigenvalues, and hence, standard random matrix theory techniques are not applicable to flux compactifications in this limit.

The absence of supersymmetric vacua near the LCS limit of general Calabi-Yau compactifications with generic fluxes is particularly relevant for studying the finiteness of the number of supersymmetric vacua [2, 4, 38-42]. Indeed, it was argued in [2] that infinite sequences of supersymmetric vacua can only occur if they accumulate in a neighbourhood of 'D-limits', which are singular points of the complex structure moduli space in which

$\left\{\vec{\Pi}, \vec{\Pi}^{*}, D_{a} \vec{\Pi}, \bar{D}_{\bar{a}} \vec{\Pi}^{*}\right\}$ degenerates and ceases to be a good symplectic basis. The LCS point is the canonical example of a 'D-limit'.

The actual existence of such an infinite sequences of vacua was left as an open question in [2], and in [39, 40] the statistical methods developed in [2] (based on the continuous flux approximation) were used to argue that no such sequence appears close to LCS points. 
Moreover, for one parameter Calabi-Yau compactifications it was shown in [42] that the neighbourhood of an LCS point contains no supersymmetric vacua, except for one sitting right at the LCS point [43].

We have here shown that the continuous flux approximation, and hence the statistical arguments of $[2,39,40]$, are not applicable for generic fluxes close to a LCS point of a general flux compactification. Nevertheless, for these generic fluxes there are no vacua (and in particularly no infinite sequence of vacua) at large complex structure, in agreement with earlier statistical studies. Our results are consistent with the type IIB vacua found in $[42,43]$ in which the cubic term in the superpotential vanishes. It would be interesting to extend our results to the non-generic case in which the superpotential is dominated by quadratic terms in the moduli.

In this paper we have considered the spectrum of moduli explicitly lifted by the flux-induced superpotential, i.e. the complex structure moduli and the axio-dilaton. It is straightforward to extend this analysis to include Kähler moduli with the no-scale Kähler potential $K_{(K)}=-2 \ln \mathcal{V}$. In type IIB compactifications, $\mathcal{V}$ is a homogeneous function of degree $3 / 2$ in the four-cycle volumes of the compactification three-fold. The corresponding Kähler moduli F-term is given by $F_{\alpha}=K_{\alpha} W$, and the scalar potential and gradient now becomes,

$$
V=4 e^{K}|W|^{2}, \quad \partial_{A} V=4 e^{K} F_{A} \bar{W},
$$

where $A$ now runs over all moduli, including the $h^{1,1}(\tilde{M})$ Kähler moduli. The spectrum of the Hessian matrix can be found following a similar method to that of section 3.1.3, and is given by,

$$
m_{i}^{2}= \begin{cases}0 & i=1, \ldots, h^{1,2}+h^{1,1}, \\ 8 m_{3 / 2}^{2} & i=h^{1,2}+h^{1,1}+1, \ldots, 2\left(h^{1,2}+h^{1,1}\right)+1, \\ 56 m_{3 / 2}^{2} & i=2\left(h^{1,2}+h^{1,1}+1\right) .\end{cases}
$$

To stabilise the Kähler moduli additional contributions, such as e.g. non-perturbative superpotential corrections, need to be included in the theory, and equation (4.2) would only give the approximate spectrum in regions of moduli space in which such corrections are small.

Our results can be used to compute the inflationary slow-roll parameters in the regions of moduli space in which (2.12) or (2.15) apply. Consistently with [1], we find that (ignoring Kähler moduli),

$$
\epsilon=\frac{1}{2}\left(\frac{2 \partial_{A} V K^{A \bar{B}} \partial_{\bar{B}} V}{V^{2}}\right)=4, \quad \eta_{\|}=\frac{\mathbf{e} \cdot \mathcal{H} \cdot \mathbf{e}}{V}=8,
$$

where e denotes the unit vector in the $\left(\partial_{A} V, \partial_{\bar{A}} V\right)^{\mathrm{T}}$ direction. Including Kähler moduli with the no-scale Kähler potential does not qualitatively change this result, as the slow-roll parameters then are given by,

$$
\epsilon=\frac{7}{4}, \quad \eta_{\|}=14
$$


We believe that these results are highly relevant for proposed attempts to construct models of axion-monodromy inflation around LCS points in the moduli space [44].

There are a number of interesting future directions of this work: while we have shown that general and typically very complicated flux compactifications exhibit universal properties in some region of the moduli space, it would be most interesting to determine if there exist classes of vacua that exhibit some universal properties as well. Moreover, it would be interesting to extend these results to other 'special points' in the moduli space, such as conifold points, Landau-Ginzburg points, and geometric engineering limits. We hope to return to these questions in future work.

\section{Acknowledgments}

We would like to thank Jose Juan Blanco-Pillado, Andreas Braun, Roberto Valandro and Timo Weigand for stimulating discussions. KS acknowledges financial support from the Spanish Consolider-Ingenio 2010 program CPAN CDS2007-00042, the Consolider EPI CSD2010-00064, the Basque Government (IT-559-10), the Spanish Ministry of Science grant (FPA 2012-34456) and by the ERC Advanced Grant 339169 "Selfcompletion". D.M. acknowledges support from a Starting Grant of the European Research Council (ERC STG Grant 279617).

Open Access. This article is distributed under the terms of the Creative Commons Attribution License (CC-BY 4.0), which permits any use, distribution and reproduction in any medium, provided the original author(s) and source are credited.

\section{References}

[1] C. Brodie and M.C.D. Marsh, The spectra of type IIB flux compactifications at large complex structure, JHEP 01 (2016) 037 [arXiv:1509.06761] [INSPIRE].

[2] S. Ashok and M.R. Douglas, Counting flux vacua, JHEP 01 (2004) 060 [hep-th/0307049] [INSPIRE].

[3] F. Denef, M.R. Douglas and B. Florea, Building a better racetrack, JHEP 06 (2004) 034 [hep-th/0404257] [INSPIRE].

[4] F. Denef and M.R. Douglas, Distributions of flux vacua, JHEP 05 (2004) 072 [hep-th/0404116] [INSPIRE].

[5] F. Denef and M.R. Douglas, Distributions of nonsupersymmetric flux vacua, JHEP 03 (2005) 061 [hep-th/0411183] [INSPIRE].

[6] D. Marsh, L. McAllister and T. Wrase, The Wasteland of Random Supergravities, JHEP 03 (2012) 102 [arXiv:1112.3034] [INSPIRE].

[7] K. Sousa and P. Ortiz, Perturbative Stability along the Supersymmetric Directions of the Landscape, JCAP 02 (2015) 017 [arXiv:1408.6521] [INSPIRE].

[8] M.C.D. Marsh, B. Vercnocke and T. Wrase, Decoupling and de Sitter Vacua in Approximate No-Scale Supergravities, JHEP 05 (2015) 081 [arXiv:1411.6625] [INSPIRE]. 
[9] A. Achucarro, P. Ortiz and K. Sousa, A new class of de Sitter vacua in String Theory Compactifications, arXiv: 1510.01273 [INSPIRE].

[10] X. Chen, G. Shiu, Y. Sumitomo and S.H.H. Tye, A global view on the search for de-Sitter vacua in (type IIA) string theory, JHEP 04 (2012) 026 [arXiv:1112.3338] [INSPIRE].

[11] U. Danielsson and G. Dibitetto, On the distribution of stable de Sitter vacua, JHEP 03 (2013) 018 [arXiv: 1212.4984] [INSPIRE].

[12] T.C. Bachlechner, D. Marsh, L. McAllister and T. Wrase, Supersymmetric vacua in random supergravity, JHEP 01 (2013) 136 [arXiv:1207.2763] [INSPIRE].

[13] D. Martinez-Pedrera, D. Mehta, M. Rummel and A. Westphal, Finding all flux vacua in an explicit example, JHEP 06 (2013) 110 [arXiv:1212.4530] [INSPIRE].

[14] T.C. Bachlechner, On Gaussian random supergravity, JHEP 04 (2014) 054 [arXiv: 1401.6187] [INSPIRE].

[15] C. Long, L. McAllister and P. McGuirk, Heavy Tails in Calabi-Yau Moduli Spaces, JHEP 10 (2014) 187 [arXiv: 1407.0709] [INSPIRE].

[16] T.D. Dimofte, Type IIB Flux Vacua at Large Complex Structure, JHEP 09 (2008) 064 [arXiv:0806.0001] [INSPIRE].

[17] D. Farquet and C.A. Scrucca, Scalar geometry and masses in Calabi-Yau string models, JHEP 09 (2012) 025 [arXiv: 1205.5728] [INSPIRE].

[18] W. Taylor and Y.-N. Wang, The F-theory geometry with most flux vacua, JHEP 12 (2015) 164 [arXiv:1511.03209] [INSPIRE].

[19] D. Gallego and M. Serone, An effective description of the landscape. I., JHEP 01 (2009) 056 [arXiv:0812.0369] [INSPIRE].

[20] D. Gallego and M. Serone, An effective description of the landscape. II, JHEP 06 (2009) 057 [arXiv: 0904.2537] [INSPIRE].

[21] L. Brizi, M. Gomez-Reino and C.A. Scrucca, Globally and locally supersymmetric effective theories for light fields, Nucl. Phys. B 820 (2009) 193 [arXiv:0904.0370] [InSPIRE].

[22] P. Binetruy, G. Dvali, R. Kallosh and A. Van Proeyen, Fayet-Iliopoulos terms in supergravity and cosmology, Class. Quant. Grav. 21 (2004) 3137 [hep-th/0402046] [INSPIRE].

[23] A. Achucarro and K. Sousa, F-term uplifting and moduli stabilization consistent with Kähler invariance, JHEP 03 (2008) 002 [arXiv:0712.3460] [INSPIRE].

[24] A. Achucarro, S. Hardeman and K. Sousa, Consistent Decoupling of Heavy Scalars and Moduli in $N=1$ Supergravity, Phys. Rev. D 78 (2008) 101901 [arXiv:0806.4364] [INSPIRE].

[25] A. Achucarro, S. Hardeman and K. Sousa, F-term uplifting and the supersymmetric integration of heavy moduli, JHEP 11 (2008) 003 [arXiv:0809.1441] [INSPIRE].

[26] T.W. Grimm and J. Louis, The effective action of $N=1$ Calabi-Yau orientifolds, Nucl. Phys. B 699 (2004) 387 [hep-th/0403067] [INSPIRE].

[27] F. Denef, Les Houches Lectures on Constructing String Vacua, in String theory and the real world: From particle physics to astrophysics. Proceedings of Summer School in Theoretical Physics, 87th Session, Les Houches, France, 2-27 July 2007, pg. 483 [arXiv:0803.1194] [INSPIRE]. 
[28] S. Gukov, C. Vafa and E. Witten, CFT's from Calabi-Yau four folds, Nucl. Phys. B 584 (2000) 69 [Erratum ibid. B 608 (2001) 477] [hep-th/9906070] [INSPIRE].

[29] S. Hosono, A. Klemm, S. Theisen and S.-T. Yau, Mirror symmetry, mirror map and applications to Calabi-Yau hypersurfaces, Commun. Math. Phys. 167 (1995) 301 [hep-th/9308122] [INSPIRE].

[30] P. Berglund et al., Periods for Calabi-Yau and Landau-Ginzburg vacua, Nucl. Phys. B 419 (1994) 352 [hep-th/9308005] [INSPIRE].

[31] S. Hosono, A. Klemm, S. Theisen and S.-T. Yau, Mirror symmetry, mirror map and applications to complete intersection Calabi-Yau spaces, Nucl. Phys. B 433 (1995) 501 [hep-th/9406055] [INSPIRE].

[32] M. Cicoli, D. Klevers, S. Krippendorf, C. Mayrhofer, F. Quevedo and R. Valandro, Explicit de Sitter Flux Vacua for Global String Models with Chiral Matter, JHEP 05 (2014) 001 [arXiv: 1312.0014] [INSPIRE].

[33] P. Candelas and X. de la Ossa, Moduli Space of Calabi-Yau Manifolds, Nucl. Phys. B 355 (1991) 455 [inSPIRE].

[34] S. Hosono, A. Klemm and S. Theisen, Lectures on mirror symmetry, Lect. Notes Phys. 436 (1994) 235 [hep-th/9403096] [INSPIRE].

[35] Y. Honma and M. Manabe, Exact Kähler Potential for Calabi-Yau Fourfolds, JHEP 05 (2013) 102 [arXiv: 1302.3760] [inSPIRE].

[36] M. Arends et al., D\%-Brane Moduli Space in Axion Monodromy and Fluxbrane Inflation, Fortsch. Phys. 62 (2014) 647 [arXiv: 1405. 0283] [INSPIRE].

[37] A. Hebecker, P. Mangat, F. Rompineve and L.T. Witkowski, Tuning and Backreaction in F-term Axion Monodromy Inflation, Nucl. Phys. B 894 (2015) 456 [arXiv:1411.2032] [INSPIRE].

[38] B.S. Acharya and M.R. Douglas, A finite landscape?, hep-th/0606212 [INSPIRE].

[39] T. Eguchi and Y. Tachikawa, Distribution of flux vacua around singular points in Calabi-Yau moduli space, JHEP 01 (2006) 100 [hep-th/0510061] [INSPIRE].

[40] G. Torroba, Finiteness of Flux Vacua from Geometric Transitions, JHEP 02 (2007) 061 [hep-th/0611002] [INSPIRE].

[41] R. Bousso and J. Polchinski, Quantization of four form fluxes and dynamical neutralization of the cosmological constant, JHEP 06 (2000) 006 [hep-th/0004134] [INSPIRE].

[42] A.P. Braun, N. Johansson, M. Larfors and N.-O. Walliser, Restrictions on infinite sequences of type IIB vacua, JHEP 10 (2011) 091 [arXiv:1108.1394] [INSPIRE].

[43] U.H. Danielsson, N. Johansson and M. Larfors, The World next door: results in landscape topography, JHEP 03 (2007) 080 [hep-th/0612222] [INSPIRE].

[44] I. García-Etxebarria, T.W. Grimm and I. Valenzuela, Special Points of Inflation in Flux Compactifications, Nucl. Phys. B 899 (2015) 414 [arXiv:1412.5537] [INSPIRE]. 